\title{
What's New in Renal Cell Cancer Research? Highlights of GU-ASCO 2015
}

Cite as: Can Urol Assoc J 2015;9(5-6-Suppl3):S154-5. http://dx.doi.org/10.5489/cuai.2995

Published online June 15, 2015.

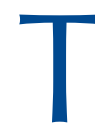

he 2015 Genitourinary Cancers Symposium (GU-ASCO 2015) included the presentation of important new research in the field of renal cell cancer (RCC). The following brief review provides key highlights of this research.

\section{No benefit from adjuvant vascular endothelial growth fac- tor (VEGF) inhibition for locally advanced RCC}

The Sunitinib Malate or Sorafenib Tosylate in Treating Patients With Kidney Cancer That Was Removed By Surgery (ASSURE) study was a Phase 3 study involving 1943 patients with resected RCC (pT1b high-grade to $\mathrm{pT} 4$ any grade) at intermediate-high to very high risk. ${ }^{1}$ Subjects were randomized to sunitinib daily for four weeks of six week cycle, sorafenib daily, or placebo, with treatment continuing for one year. The primary endpoint was disease-free survival (DFS). ${ }^{1}$

There was no benefit for either adjuvant treatment relative to placebo over a median follow-up of approximately six years, nor was there any benefit in overall survival (OS) (Fig. 1). The authors concluded that adjuvant VEGF inhibition with sunitinib and sorafenib be abandoned as a potential therapy for locally advanced RCC.

\section{Potential benefit of combined VEGF inhibition sunitinib and cytotoxic chemotherapy for rapidly progressive RCC}

A Phase 2 study involving 72 patients with sarcomatoid and/or poor-risk metastatic RCC evaluated the combination of sunitinib (37.5 mg daily, two-weeks on, one-week off) and gemcitabine $\left(1,000 \mathrm{mg} / \mathrm{m}^{2}\right.$ IV day 1 and 8 of every 21 -day cycle) in a single-arm design. ${ }^{2}$ The primary endpoint of objective response rate (defined by Response Evaluation Criteria In Solid Tumors [RECIST] criteria) was achieved by $26 \%(\mathrm{n} / \mathrm{N}=10 / 32)$ of evaluable patients. The stable disease rate was $38 \%$. This combination is under further investigation in a prospective, randomized trial.

\section{Everolimus superior to dual TORC1 $/ 2$ inhibitor for meta- static, VEGF-refractory RCC}

This Phase 2 study included 49 patients with VEGF-refractory metastatic RCC. ${ }^{3}$ These subjects were randomized to receive the dual TORC1/2 inhibitor AZD2014 or the isolated TORC1 inhibitor everolimus. Median progression-free survival (PFS) with everolimus was 4.6 months, compared to 1.8 months for patients receiving AZD2014. Similarly, median OS at final analysis was 16.7 months with everolimus and 6.2 months with AZD2014. Thus, for both progression-free survival (PFS) and OS, everolimus therapy was found to be superior to AZD2014.

\section{Similar efficacy of everolimus and axitinib as second-line therapy for metastatic RCC}

In this retrospective study, investigators compared the use of second-line everolimus and axitinib for metastatic RCC following tyrosine kinase inhibitor (TKI) therapy.

This study was a chart review of 298 patients treated with everolimus and 122 treated with axitinib in the United States; charts were recruited from a national panel of medical oncologists or hematologists/oncologists.

In this study, there were no significant between-group differences detected for OS or PFS.

\section{Correlation between toxicity and efficacy with everolimus}

This study was designed to assess the association between everolimus-related toxicity requiring discontinuation or dose reduction and efficacy outcomes. ${ }^{5}$ The data come from a retrospective review of 79 patients with metastatic RCC at two Italian centres.

The OS rate among those patients with stomatitis-cutaneous toxicity events (SCTE) associated with everolimus treatment was significantly better than among those with no such toxicity $(p=0.004)$ (Fig. 2). PFS was also significantly longer among those with SCTE $(p=0.047)$. 


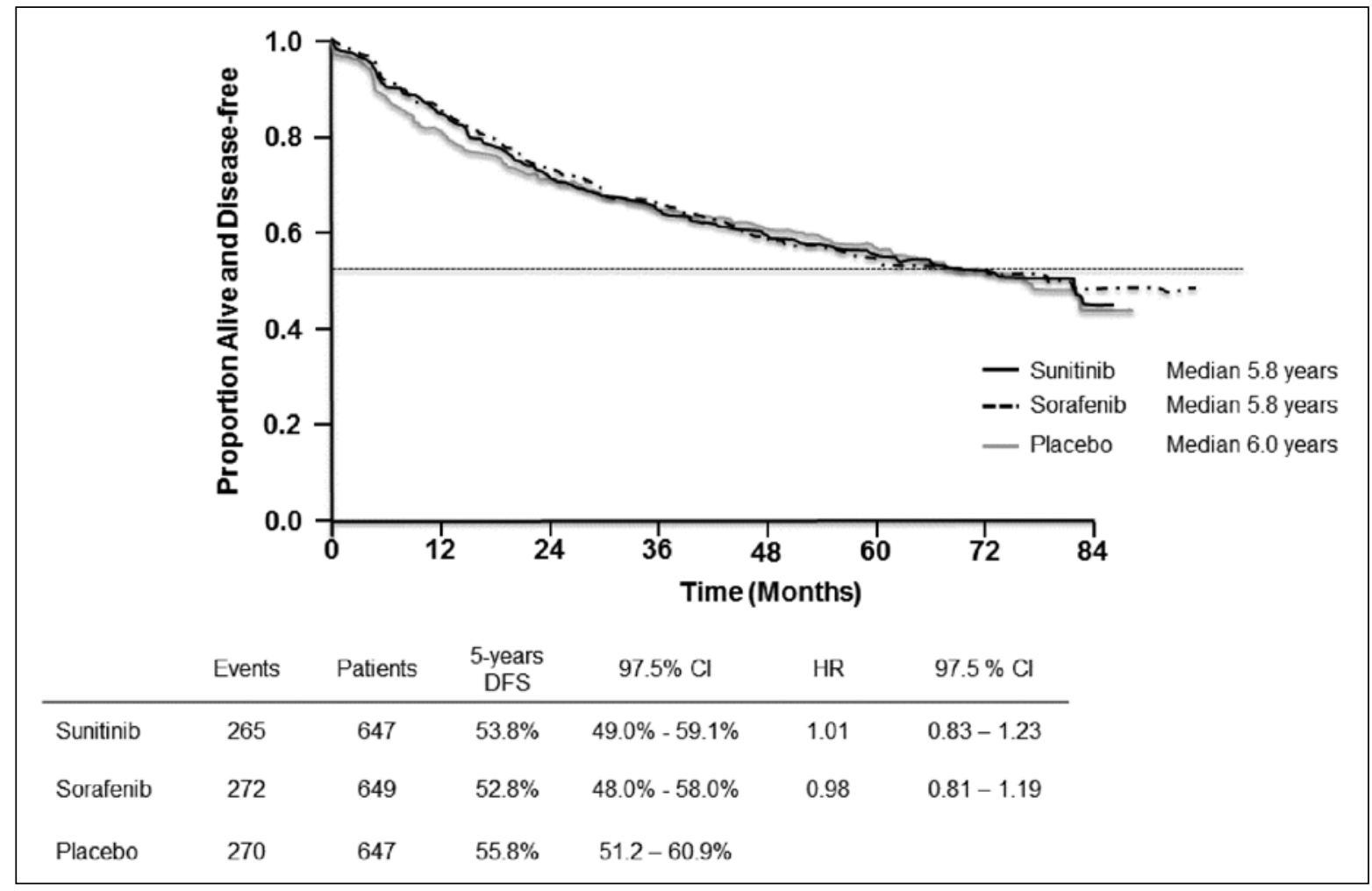

Fig. 1. No benefit from adjuvant sunitinib or sorafenib in locally advanced renal cell carcinoma (ASSURE).

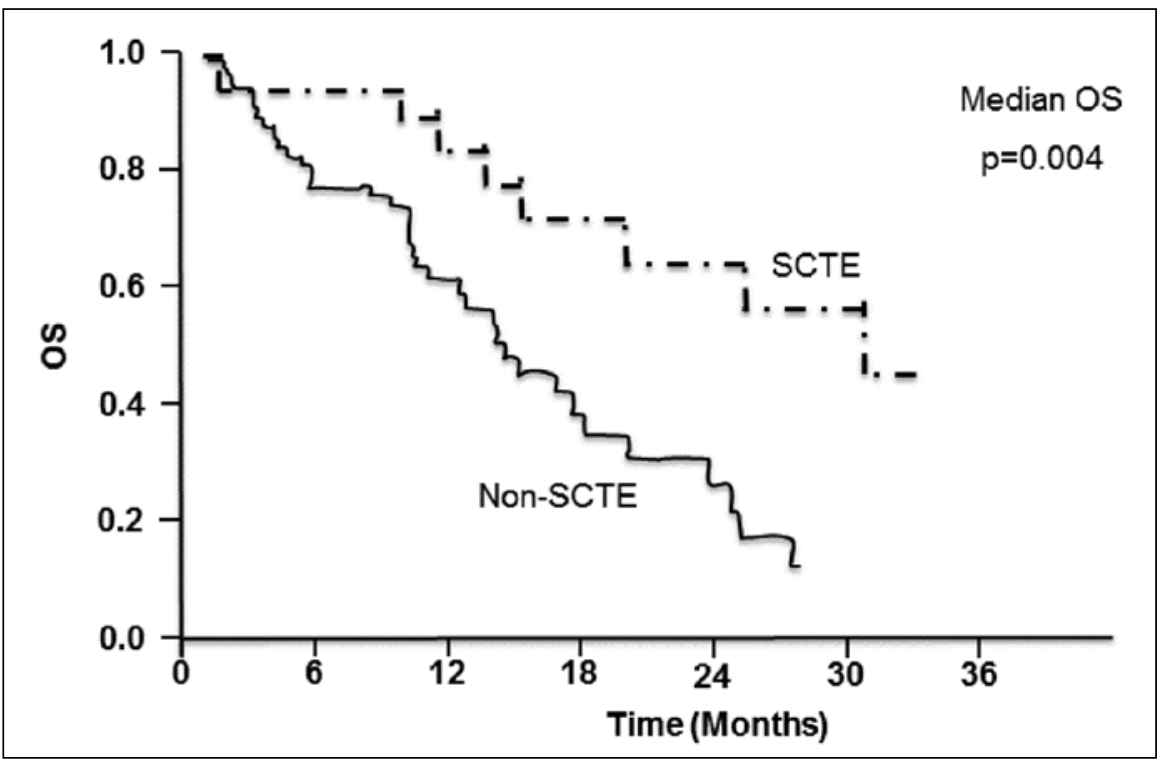

Fig. 2. Stomatitis-cutaneous toxicity events are associated with improved overall survival with everolimus therapy.

\section{References}

1. Haas NB et al. Initial results from ASSURE (E2805): Adjuvant sorafenib or sunitinib for unfavorable renal carcinoma, an ECOG-ACRIN-led, NCTN phase III trial. Prese I Clin Oncol 2015; 33(suppl 7; abstr 403).

2. McKay RR et al. A phase II trial of sunitinib and gemcitabine in sarcomatoid and/or poor-risk patients with metastatic renal cell carcinoma. J Clin Oncol 2015; 33(suppl 7; abstr 408).

3. Powles T et al. A randomized phase II study of AZ2014 versus everolimus in patients with VEGF refractory metastatic clear cell renal cancer (mRCC). J Clin Oncol 2015; 33(suppl 7; abstr 409).
4. Vogelzang NJ et al. Comparative effectiveness of everolimus (EVE) and axitinib (AXI) for second-line treatment of metastatic renal cell carcinoma (mRCC) in the United States: A retrospective chart review. J Clin Oncol 2015; 33(suppl 7; abstr 500).

5. Burgio SL et al. Correlation between everolimus discontinuation or dose reduction induced by stomatitis or cutaneous toxicity and clinical outcome in patients with metastatic renal cell carcinoma. J Clin Oncol 2015; 33(suppl 7; abstr 448). 


\title{
Funding in the form of an educational grant was provided by
}

Astellas Pharma Canada, Inc.

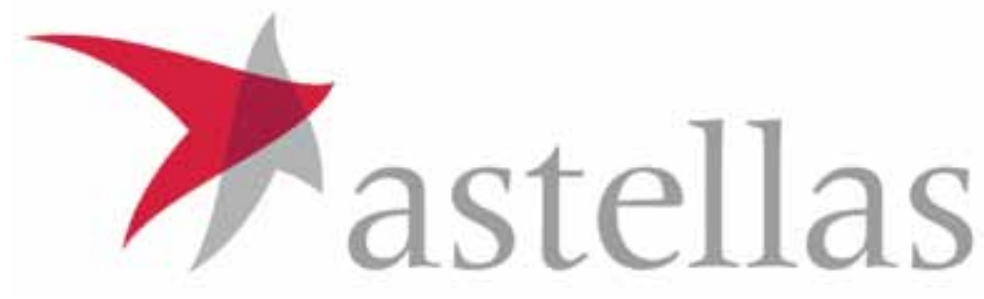

\author{
and
}

Novartis Pharmaceuticals Canada, Inc.

(1) NOVARTIS 